\title{
Expressions for the threshold current of multipass beam breakup in recirculating linacs from single cavity models
}

\author{
Byung C. Yunn \\ Thomas Jefferson National Accelerator Facility, Newport News, Virginia 23606, USA
}

(Received 21 May 2005; published 4 October 2005)

\begin{abstract}
We investigate multipass beam breakup (BBU) in a recirculating linear accelerator in the framework of a single cavity model. We present expressions for the beam breakup threshold current for various situations derived from a perturbative solution of BBU equations. These formulae should serve as a guide to understand the BBU phenomenon for a particular system and also as a tool to estimate the BBU threshold current quickly. Many of the results presented are more general than previous considerations because they include the effects of coupling between the two transverse polarizations in each dipole higher order mode.
\end{abstract}

DOI: 10.1103/PhysRevSTAB.8.104401

PACS numbers: 29.27.Bd, 41.85.Ja, 52.35. $-\mathrm{g}$

\section{INTRODUCTION}

Recently multipass beam breakup (BBU) was observed at the Jefferson Laboratory (JLAB) Free Electron Laser (FEL) Upgrade [1]. As part of an effort to operate the accelerator at a beam current above the observed beam breakup threshold a rotation of the transverse planes was introduced to intentionally couple the horizontal $(x)$ and vertical $(y)$ beam motion. In a decoupled machine, BBU occurs when an $x$-deflecting higher order mode (HOM) causes a transverse displacement on a higher pass which can then further excite the wake which caused the $x$ impulse initially. The idea behind the rotator [2] is to break the feedback by turning an horizontal impulse to a vertical offset thereby reducing the coupling to the wake. However, one should note that a dipole HOM always has two polarizations. Unless one eliminates the other polarization completely, which is impossible in practice, a chain of feedback mechanisms will be reestablished through the coupled beam motion. Recently, we have studied this chain of beam motion and found a beam breakup threshold formula which should be useful in understanding this method of implementing a rotator to overcome the beam breakup problem in a recirculating accelerator [3]. In addition we have studied a few other common recirculating machine configurations and found useful BBU threshold formulas to be used for estimating BBU threshold currents. We present them here for the benefit of accelerator designers in the future.

\section{FORMULATION OF THE BBU PROBLEM IN RECIRCULATING LINACS}

Simply stated, multipass BBU occurs because dipole wake fields in cavities can grow exponentially in strength due to the feedback loop formed by the recirculating bunches when the average current exceeds a certain threshold value. A mathematical model for this mechanism is described below. For simplicity, in this paper we study the BBU problem in the framework of a single cavity model.
A dipole mode in a cavity always comes in two polarizations. Let the $x$ and $y$ polarization component of the delta functional wake potential of a single dipole HOM be

$$
G_{x, y}(t)=\left(\frac{c}{2}\right)\left(\frac{Z^{\prime \prime} T^{2}}{Q}\right)_{x, y} \exp \left(-\frac{\omega_{x, y} t}{2 Q_{x, y}}\right) \sin \omega_{x, y} t
$$

for negative time $t$. From causality, the functions $G_{x}(t)$ and $G_{y}(t)$ are zero for positive time. For convenience the most frequently appearing symbols and notations in this paper are defined in Table I.

We now assume that a single dipole mode can be excited in the cavity (adding more modes to the model is straightforward but not necessary for our purpose) and let $W_{x}(t)$ and $W_{y}(t)$ be accumulated dipole wakes in the cavity at time $t$. A bunch passing the cavity at time $t$ receives momentum kicks due to the wake fields excited in the cavity and the momentum at the exit of the cavity is

$$
\Delta p_{x, y}(t)=\frac{e}{c} W_{x, y}(t)
$$

When the same bunch enters the cavity after the recirculation period $t_{\tau}$, the bunch will possess dipole moments originated from the momentum kicks received on the previous pass. Thus, the bunch dipole moments at the cavity entrance on the recirculated pass are given by

$$
\begin{aligned}
& D_{x}(t)=\frac{N_{e} r_{0}}{\gamma}\left[M_{12} W_{x}\left(t-t_{\tau}\right)+M_{14} W_{y}\left(t-t_{\tau}\right)\right], \\
& D_{y}(t)=\frac{N_{e} r_{0}}{\gamma}\left[M_{32} W_{x}\left(t-t_{\tau}\right)+M_{34} W_{y}\left(t-t_{\tau}\right)\right] .
\end{aligned}
$$

These will contribute to the dipole wakes by an amount proportional to the dipole moments according to

$$
\begin{aligned}
& W_{x}(t)=\int_{-\infty}^{t} G_{x}\left(t-t^{\prime}\right) D_{x}\left(t^{\prime}\right) d t^{\prime}, \\
& W_{x}(t)=\int_{-\infty}^{t} G_{y}\left(t-t^{\prime}\right) D_{y}\left(t^{\prime}\right) d t^{\prime} .
\end{aligned}
$$


TABLE I. Summary of notations.

\begin{tabular}{|c|c|}
\hline Symbol & Definition and/or comment \\
\hline$e$ & Electron charge \\
\hline$c$ & The velocity of light \\
\hline$r_{0}$ & The radius of electron \\
\hline$N_{e}$ & The total number of electrons in a single bunch \\
\hline$E$ & $\begin{array}{l}\text { Nominal beam energy and } E=\gamma m c^{2} \text { with the relativistic } \gamma \text { factor. Note that } E \text { is not the final beam energy, rather the energy of beam in } \\
\text { the recirculation arc for a two pass machine. }\end{array}$ \\
\hline$E_{i}$ & Beam energy after the completion of the $i$ th pass through accelerating cavity \\
\hline$t_{\tau}$ & Recirculation time period of a two pass machine \\
\hline$t_{\tau}^{i j}$ & Recirculation time period from the $i$ th pass to the $j$ th pass in an $N_{p}$ pass machine. \\
\hline$t_{0}$ & The bunching period (in this paper we assume continuous wave beam operation) \\
\hline$\omega_{0}$ & The bunching frequency (i.e. $2 \pi / t_{0}$ ) \\
\hline$\omega$ & Frequency of a dipole mode \\
\hline$\omega_{x}, \omega_{y}$ & Frequency of an $x$-polarization ( $y$-polarization) component of a dipole mode (used when they are not degenerate) \\
\hline$Q$ & Quality factor of a dipole mode \\
\hline \multirow{2}{*}{$\begin{array}{l}Q_{x}, Q_{y} \\
Z^{\prime \prime} T^{2} / Q\end{array}$} & Quality factor of an $x$-polarization ( $y$-polarization) component of a dipole mode when different \\
\hline & $\begin{array}{l}\text { Shunt impedance of a dipole mode defined as }(R / Q) \text { measured at a beam radial offset divided by the square of the offset. For a dipole } \\
\text { mode this quantity does not depend on the offset. }\end{array}$ \\
\hline$\left(Z^{\prime \prime} T^{2} / Q\right)_{x, y}$ & Shunt impedance of an $x$-polarization ( $y$-polarization) component of a dipole mode (used when they have different strengths) \\
\hline$M_{i j}$ & $(i, j)$ are elements of the 4-by-4 transfer matrix $M$ for a given beam transport line using the program TRANSPORT units $\left(x, x^{\prime}, y, y^{\prime}\right)$ \\
\hline$\left(M^{i j}\right)_{12}$ & $\begin{array}{l}(1,2) \text { element of the beam transfer matrix } M^{i j} . M^{i j} \text { is defined as the transfer matrix for a beamline stretching from a cavity location at the } \\
i \text { th pass to the same cavity location at the } j \text { th pass of the beam. Used when } N_{p}>2 \text {. }\end{array}$ \\
\hline
\end{tabular}

Consequently, we are left to solve the following coupled integral equations for the wake functions

$$
\begin{aligned}
W_{x}(t)= & \frac{N_{e} r_{0}}{\gamma} \int_{-\infty}^{t} G_{x}\left(t-t^{\prime}\right)\left[M_{12} W_{x}\left(t^{\prime}-t_{r}\right)\right. \\
& \left.+M_{14} W_{y}\left(t^{\prime}-t_{\tau}\right)\right] d t^{\prime}, \\
W_{y}(t)= & \frac{N_{e} r_{0}}{\gamma} \int_{-\infty}^{t} G_{x}\left(t-t^{\prime}\right)\left[M_{32} W_{x}\left(t^{\prime}-t_{r}\right)\right. \\
& \left.+M_{34} W_{y}\left(t^{\prime}-t_{\tau}\right)\right] d t^{\prime} .
\end{aligned}
$$

Now let us consider the case of an $N_{p}$ pass recirculating linac in detail. Since the beam consists of an infinite train of bunches separated by the bunching period $t_{0}$, the total dipole moments the beam possesses at the cavity location are

$$
\begin{aligned}
D_{x}(t)= & \frac{N_{e} r_{0}}{\gamma}\left[\sum_{j>i=1}^{N_{p}} \frac{E}{E_{i}}\left(M^{i j}\right)_{12} W_{x}\left(t-t_{\tau}^{i j}\right)\right. \\
& \left.+\sum_{j>i=1}^{N_{p}} \frac{E}{E_{i}}\left(M^{i j}\right)_{14} W_{y}\left(t-t_{\tau}^{i j}\right)\right] \sum_{m=-\infty}^{\infty} \delta\left(t-m t_{0}\right),
\end{aligned}
$$

$$
\begin{aligned}
D_{y}(t)= & \frac{N_{e} r_{0}}{\gamma}\left[\sum_{j>i=1}^{N_{p}} \frac{E}{E_{i}}\left(M^{i j}\right)_{32} W_{x}\left(t-t_{\tau}^{i j}\right)\right. \\
& \left.+\sum_{j>i=1}^{N_{p}} \frac{E}{E_{i}}\left(M^{i j}\right)_{34} W_{y}\left(t-t_{\tau}^{i j}\right)\right] \sum_{m=-\infty}^{\infty} \delta\left(t-m t_{0}\right) .
\end{aligned}
$$

Inserting Eqs. (9) and (10) into Eqs. (5) and (6), we obtain coupled BBU equations for an $N_{p}$ pass machine,

$$
\begin{aligned}
W_{x}(t)= & \frac{N_{e} r_{0}}{\gamma} \\
& \times\left\{\begin{array}{c}
\sum_{j>i=1}^{N} \frac{E}{E_{i}}\left(M^{i j}\right)_{12} \sum_{m} G_{x}\left(t-m t_{0}\right) W_{x}\left(m t_{0}-t_{\tau}^{i j}\right) \\
+\sum_{j>i=1}^{N} \frac{E}{E_{i}}\left(M^{i j}\right)_{14} \sum_{m} G_{x}\left(t-m t_{0}\right) W_{y}\left(m t_{0}-t_{\tau}^{i j}\right)
\end{array}\right\}, \\
W_{y}(t)= & \frac{N_{e} r_{0}}{\gamma} \\
& \times\left\{\begin{array}{c}
\sum_{j>i=1}^{N} \frac{E}{E_{i}}\left(M^{i j}\right)_{32} \sum_{m} G_{y}\left(t-m t_{0}\right) W_{x}\left(m t_{0}-t_{\tau}^{i j}\right) \\
\left.+\sum_{j>i=1}^{N} \frac{E}{E_{i}}\left(M^{i j}\right)_{34} \sum_{m} G_{y}\left(t-m t_{0}\right) W_{y}\left(m t_{0}-t_{\tau}^{i j}\right)\right\} .
\end{array}\right.
\end{aligned}
$$

These equations will be simplified appropriately for several specific cases which we will consider in the following sections. First, we note the following useful relation for the summation of wakes over beam bunches which will be frequently used:

$$
\sum_{m=-\infty}^{\infty} e^{i \Omega\left(m t_{0}-t_{\tau}\right)} G_{x}\left(t-m t_{0}\right)=\frac{c}{2}\left(Z^{\prime \prime} T^{2} / Q\right)_{x} e^{i \Omega\left(t-t_{\tau}\right)} H\left(\Omega, \omega_{x}\right),
$$

where

$$
H(\Omega, \omega)=\frac{\xi \sin \omega t_{0}}{1+\xi^{2}-2 \xi \cos \omega t_{0}},
$$


and

$$
\xi=\exp \left(-\frac{\omega}{2 Q} t_{0}-i \Omega t_{0}\right)
$$

\section{TWO PASS MACHINE: UNCOUPLED TRANSVERSE BEAM OPTICS}

A model for BBU using a single cavity with one dominating dipole mode has proven to be an accurate approximation for the JLAB IR FEL Upgrade [4]. With no $x-y$ coupling in the transverse beam motion this is the simplest case to handle.

We specifically consider beam behavior in the $x$ plane. The BBU equation in this case is

$$
W_{x}(t)=\frac{N_{e} r_{0}}{\gamma} M_{12} \sum_{m} G_{x}\left(t-m t_{0}\right) W_{x}\left(m t_{0}-t_{\tau}\right) .
$$

Searching for a harmonic solution for the wake of the form $W_{x}=A \exp (i \Omega t)$ we find that the frequency must satisfy the following relation

$$
e^{i \Omega t_{\tau}}=\frac{\pi e I}{\gamma m c \omega_{0}}\left(\frac{Z^{\prime \prime} T^{2}}{Q}\right)_{x} M_{12} H\left(\Omega, \omega_{x}\right) .
$$

Numerically, solutions to Eq. (17) can be found by determining the current $I$ as a function of real $\Omega$ while scanning in frequency. In general the current $I$ turns out to be complex except for a few isolated real frequency values and the smallest positive $I$ among them is the threshold current for BBU.

Even though Eq. (17) appears to be very simple in form, the solution for $\Omega$ as a function of the current is not available analytically in general (two special cases where an analytical solution is obtainable are treated in Appendix A). However, there are at least two ways (we can imagine) to analyze the present problem in order to get an expression for the threshold current. One method would be to equate the energy that the wake field dissipates with the energy induced by a bunch dipole moment during a single bunching period, noting that an equilibrium state can be maintained if the net value is zero. As the dipole moment is proportional to the average current this will occur at the current value identified as the threshold current for instability. Another approach would be a perturbative solution of $\Omega$ in $I$. This is the method we will use throughout the paper.

Let us start by first introducing an expansion parameter for our perturbative solution,

$$
\varepsilon=\frac{\pi e I}{\gamma m c \omega_{0}}\left(\frac{Z^{\prime \prime} T^{2}}{Q}\right)_{x} M_{12}
$$

and assume $\varepsilon$ is small and $\Omega=\alpha+\beta \varepsilon$. Inserting the assumed solution into Eq. (17) and expanding terms in $\varepsilon$ (which is justified because of our assumption) and keeping terms only to first order, we find

$$
\alpha=\mp \omega_{x}+\frac{i}{2 Q_{x}} \omega_{x} \quad \text { and } \quad \beta= \pm \frac{1}{2 t_{0}} \exp \left(-i \alpha t_{\tau}\right) \text {. }
$$

It follows that

$$
\begin{aligned}
& \operatorname{Re} \Omega=\mp \omega_{x} \pm \frac{\varepsilon}{2 t_{0}} e^{\omega_{x} t_{\tau} / 2 Q_{x}} \cos \omega_{x} t_{\tau} \quad \text { and } \\
& \operatorname{Im} \Omega=\frac{\omega_{x}}{2 Q_{x}}\left(1+\frac{\varepsilon Q_{x}}{\omega_{x} t_{0}} e^{\omega_{x} t_{\tau} / 2 Q_{x}} \sin \omega_{x} t_{\tau}\right) .
\end{aligned}
$$

The threshold current $I_{\text {th }}$ is found from the condition $\operatorname{Im} \Omega=0$ at $I=I_{\text {th }}$. Applying this condition we find the first order formula for the BBU threshold current to be

$$
I_{\mathrm{th}}=-\frac{2 E \omega_{x}}{e c\left(Z^{\prime \prime} T^{2} / Q\right)_{x} Q_{x} M_{12} e^{\omega_{x} t_{\tau} / 2 Q_{x}} \sin \omega_{x} t_{\tau}} .
$$

We note that this formula has been known for quite some time [5].

Expressing $\varepsilon$ in terms of $I_{\text {th }}$ one indeed finds that our assumption that $\varepsilon$ is being small is satisfied in most cases. A notable exception is the case where $\sin \omega_{x} t_{\tau}$ is close to zero. In such cases we need to carry out an expansion in $\varepsilon$ to second order as the first order formula is clearly inadequate providing an infinitely large threshold current value. Performing the straightforward, but tedious, calculations we find for the expansion of $\Omega=\alpha+\beta \varepsilon+\delta \varepsilon^{2}$

$$
\delta=\mp \exp \left(-2 i \alpha t_{\tau}\right) \frac{1}{4 t_{0}}\left(\frac{\cot \omega t_{0}}{2} \pm i \frac{t_{\tau}+2 t_{0}}{t_{0}}\right),
$$

with $\alpha, \beta$ as in Eq. (19).

Consequently, we are led to the following second order formula:

$$
I_{\mathrm{th}}=\frac{2 E\left[\sin \omega_{x} t_{\tau} \mp \sqrt{\sin ^{2} \omega_{x} t_{\tau}+\left(2 \omega_{x} / Q_{x}\right) \bar{\Delta}}\right]}{e c\left(Z^{\prime \prime} T^{2} / Q\right)_{x} M_{12} e^{\omega_{x} t_{\tau} / 2 Q_{x}} \Delta},
$$

where

$$
\begin{aligned}
\Delta= & \frac{t_{0}}{2}\left\{\left(1-\sin \omega_{x} t_{0}\right) \cos 2 \omega_{x} t_{\tau}-\sin \omega_{x}\left(2 t_{\tau}+t_{0}\right)\right\} \\
& +t_{\tau} \cos ^{2} \omega_{x} t_{\tau} .
\end{aligned}
$$

For the case of a mode oriented in the $y$ direction $M_{12}$ should be replaced by $M_{34}$ in Eqs. (16)-(18), (21), and (23). Note that the first order formula can be obtained from the second order formula when $\sin ^{2} \omega t_{\tau} \gg 2 \omega \Delta / Q$, which clearly shows the limit of validity for using Eq. (21).

\section{IV. $N>2$ PASS MACHINE: UNCOUPLED TRANSVERSE BEAM OPTICS}

For the case of a machine with more than 2 passes, Eqs. (11) and (12) become 


$$
W_{x}(t)=\frac{N_{e} r_{0}}{\gamma} \sum_{j>i=1}^{N} \frac{E}{E_{i}}\left(M^{i j}\right)_{12} \sum_{m} G_{x}\left(t-m t_{0}\right) W_{x}\left(m t_{0}-t_{\tau}^{i j}\right),
$$

$$
W_{y}(t)=\frac{N_{e} r_{0}}{\gamma} \sum_{j>i=1}^{N} \frac{E}{E_{i}}\left(M^{i j}\right)_{34} \sum_{m} G_{y}\left(t-m t_{0}\right) W_{y}\left(m t_{0}-t_{\tau}^{i j}\right) .
$$

As one can see, for more than one recirculation, the relevant machine parameters for the model quickly multiply. Starting with Eq. (25) and following the steps leading to the BBU threshold current formula in the previous section, we easily find the following expression for the threshold current in a machine for more than one recirculation

$$
I_{\mathrm{th}}=-\frac{2 E \omega_{x}}{e c\left(Z^{\prime \prime} T^{2} / Q\right)_{x} Q_{x} \sum_{j>i=1}^{N}\left(E / E_{i}\right)\left(M^{i j}\right)_{12} e^{\omega_{x} t_{\tau} / 2 Q_{x}} \sin \omega_{x} t_{\tau}^{i j}} .
$$

The BBU threshold for the case of a $y$ polarization is readily obtained from Eq. (27) by replacing all $x$ quantities with corresponding $y$ quantities.

The accuracy and the usefulness of estimated threshold current information provided by Eqs. (21) and (27) has been tested extensively against results of numerical codes though not well documented at JLAB. However, we refer to Ref. [6] for an example of a comparison study showing a nice agreement (especially, see Figs. 2-5). A further study on the accuracy of threshold current predictions from Eq. (21) is in Appendix B.

\section{TWO PASS MACHINE: COUPLED TRANSVERSE BEAM OPTICS}

\section{A. Symmetric case}

It is simple matter to derive the BBU equation for the case of coupled optics for a two pass machine from the general result of Sec. II. We find that Eqs. (11) and (12) become

$$
\begin{aligned}
W_{x}(t)= & \frac{N_{e} r_{0}}{\gamma}\left\{M_{12} \sum_{m} G_{x}\left(t-m t_{0}\right) W_{x}\left(m t_{0}-t_{\tau}\right)\right. \\
& \left.+M_{14} \sum_{m} G_{x}\left(t-m t_{0}\right) W_{y}\left(m t_{0}-t_{\tau}\right)\right\}, \\
W_{y}(t)= & \frac{N_{e} r_{0}}{\gamma}\left\{M_{32} \sum_{m} G_{y}\left(t-m t_{0}\right) W_{x}\left(m t_{0}-t_{\tau}\right)\right. \\
& \left.+M_{34} \sum_{m} G_{y}\left(t-m t_{0}\right) W_{y}\left(m t_{0}-t_{\tau}\right)\right\} .
\end{aligned}
$$

Assuming solutions of the form $W_{x}=A_{x} \exp (i \Omega t)$ and $W_{y}=A_{y} \exp (i \Omega t)$ we get a coupled BBU equation in frequency space,

$$
\left(\begin{array}{l}
A_{x} \\
A_{y}
\end{array}\right)=\frac{\pi e I}{\gamma m c \omega_{0}} \exp \left(-i \Omega t_{\tau}\right)\left(\begin{array}{ll}
M_{12}\left(Z^{\prime \prime} T^{2} / Q\right)_{x} H\left(\Omega, \omega_{x}\right) & M_{14}\left(Z^{\prime \prime} T^{2} / Q\right)_{x} H\left(\Omega, \omega_{x}\right) \\
M_{32}\left(Z^{\prime \prime} T^{2} / Q\right)_{y} H\left(\Omega, \omega_{y}\right) & M_{34}\left(Z^{\prime \prime} T^{2} / Q\right)_{y} H\left(\Omega, \omega_{y}\right)
\end{array}\right)\left(\begin{array}{l}
A_{x} \\
A_{y}
\end{array}\right) .
$$

Now we consider the case of a single dipole mode with two polarizations of identical strength. We also assume that we can arrange the beam optics such that $M_{12}=M_{34}=0$ with a rotator, for example, as has been suggested by Rand and Smith [2]. The problem in this instance reduces to solving

$$
\begin{aligned}
\left(\begin{array}{c}
A_{x} \\
A_{y}
\end{array}\right)= & \frac{\pi e I}{\gamma m c \omega_{0}}\left(\frac{Z^{\prime \prime} T^{2}}{Q}\right) H(\Omega, \omega) \exp \left(-i \Omega t_{\tau}\right) \\
& \times\left(\begin{array}{cc}
0 & M_{14} \\
M_{32} & 0
\end{array}\right)\left(\begin{array}{l}
A_{x} \\
A_{y}
\end{array}\right) .
\end{aligned}
$$

Equation (29) leads to

$$
e^{i \Omega t_{\tau}}= \pm \frac{\pi e I}{\gamma m c \omega_{0}}\left(\frac{Z^{\prime \prime} T^{2}}{Q}\right) \sqrt{M_{14} M_{32}} H(\Omega, \omega) .
$$

Comparing Eq. (32) with Eq. (18) one can almost write down an approximate solution of the frequency $\Omega$. However, there is a new feature in the present case.
Namely, since the product $M_{14} M_{32}$ can become negative our perturbative expansion parameter can also be purely imaginary. When $M_{14} M_{32}<0$, Eq. (20) should be replaced by

$$
\begin{aligned}
& \operatorname{Re} \Omega=\mp \omega_{x} \pm \frac{\varepsilon}{2 t_{0}} e^{\omega_{x} t_{\tau} / 2 Q_{x}} \cos \omega_{x} t_{\tau} \quad \text { and } \\
& \operatorname{Im} \Omega=\frac{\omega_{x}}{2 Q_{x}}\left(1 \pm \frac{\varepsilon Q_{x}}{\omega_{x} t_{0}} e^{\omega_{x} t_{\tau} / 2 Q_{x}} \cos \omega_{x} t_{\tau}\right) .
\end{aligned}
$$

As a result the usual $\sin \omega_{x} t_{\tau}$ term appearing in the denominator of threshold formula needs to be replaced by $\cos \omega_{x} t_{\tau}$. Keeping that in mind and applying the steps that led to the threshold expression in Sec. II [Eqs. (17) and (21)] we find

$$
\begin{aligned}
& I_{\text {th }}=\frac{2 E \omega}{e c\left(Z^{\prime \prime} T^{2} / Q\right) Q \sqrt{M_{14} M_{32}} e^{\omega t_{\tau} / 2 Q}\left|\sin \omega t_{\tau}\right|} \\
& \quad \text { for } M_{14} M_{32}>0
\end{aligned}
$$


and

$$
I_{\text {th }}=\frac{2 E \omega}{e c\left(Z^{\prime \prime} T^{2} / Q\right) Q \sqrt{-M_{14} M_{32}} e^{\omega t_{\tau} / 2 Q}\left|\cos \omega t_{\tau}\right|}
$$$$
\text { for } M_{14} M_{32}<0 \text {. }
$$

The prediction of Eqs. (34) and (35) was shown to agree very well with a numerical analysis [7].

\section{B. Asymmetric case}

In the case of two asymmetric polarizations, new features appear reflecting the change in physics of driven coupled resonators. Assuming the optics of $M_{12}=M_{34}=$ 0 with a rotator as in the previous subsection, we need to solve the following equation:

$$
e^{i \Omega t_{\tau}}= \pm \frac{\pi e I}{\gamma m c \omega_{0}} \sqrt{\left(Z^{\prime \prime} T^{2} / Q\right)_{x}\left(Z^{\prime \prime} T^{2} / Q\right)_{y}} \sqrt{M_{14} \bar{M}_{32}} \sqrt{H\left(\Omega, \omega_{x}\right) H\left(\Omega, \omega_{y}\right)} .
$$

We square both sides of Eq. (36) and define an expansion parameter

$$
\varepsilon \equiv \frac{\pi e I}{\gamma m c \omega_{0}} \sqrt{\left(Z^{\prime \prime} T^{2} / Q\right)_{x}\left(Z^{\prime \prime} T^{2} / Q\right)_{y}} \sqrt{M_{14} M_{32}}
$$

and then expand $\Omega$ keeping terms to first order in $\varepsilon^{2}$.

After straightforward computations we find that the BBU threshold current is determined by the smaller of the two current values when they are both positive from the expression

$$
I_{\mathrm{th}}=\sqrt{\frac{2}{\pi}} \frac{E \sqrt{\omega_{0} \omega_{x}} \exp \left(\frac{1}{2}\left[\left(\omega_{y} / 2 Q_{y}\right)-\left(\omega_{x} / 2 Q_{x}\right)\right] t_{0}\right)}{e c \sqrt{\left(Z^{\prime \prime} T^{2} / Q\right)_{y}\left(Z^{\prime \prime} T^{2} / Q\right)_{x} Q_{x}} e^{\omega_{x} t_{\tau} / 2 Q_{x}} \sqrt{-M_{14} M_{32} \sin \omega_{x}\left(2 t_{\tau}+t_{0}\right) \sin \omega_{y} t_{0}}},
$$

and a similar expression for $I_{\text {th }}$ with $x$ an $y$ replaced from Eq. (37). Note that now the bunching frequency plays a prominent role in determining the threshold current.

\section{Rotated polarization}

It may happen that two polarization components of a dipole mode may not align exactly with the $x$ and $y$ axis of the coordinate system set up for the accelerator. Consider the case where the $x$ polarization is rotated counterclockwise by an angle $\alpha$ about the positive $z$ axis [8].

This case requires special attention as the BBU equations cannot be read off directly from our master equations in Sec. II since these equations are derived assuming $\alpha=0$ (i.e., no rotation). Let us start with momentum kicks re- ceived by the bunch

$$
\begin{aligned}
& \Delta p_{x}(t)=\frac{e}{c}\left[W_{x}(t) \cos \alpha-W_{y}(t) \sin \alpha\right], \\
& \Delta p_{y}(t)=\frac{e}{c}\left[W_{x}(t) \sin \alpha-W_{y}(t) \cos \alpha\right] .
\end{aligned}
$$

These momentum kicks are converted into position offsets at the cavity in the process of recirculating the beam. Position offsets multiplied by the total bunch charge then provide the dipole moments of the bunch in our model. Induced dipole moments will excite more wake fields which may strengthen existing wakes thus facilitating the instability. For induced dipole moments at time $t$ we obtain

$$
\begin{aligned}
& D_{x}(t)=\frac{N_{e} r_{0}}{\gamma}\left\{\begin{array}{c}
\left(M_{12} \cos \alpha+M_{32} \sin \alpha\right)\left[W_{x}\left(t-t_{\tau}\right) \cos \alpha-W_{y}\left(t-t_{\tau}\right) \sin \alpha\right] \\
+\left(M_{14} \cos \alpha+M_{34} \sin \alpha\right)\left[W_{x}\left(t-t_{\tau}\right) \sin \alpha+W_{y}\left(t-t_{\tau}\right) \cos \alpha\right]
\end{array}\right] \sum_{m} \delta\left(t-m t_{0}\right), \\
& D_{y}(t)=\frac{N_{e} r_{0}}{\gamma}\left\{\begin{array}{c}
\left(M_{32} \cos \alpha-M_{12} \sin \alpha\right)\left[W_{x}\left(t-t_{\tau}\right) \cos \alpha-W_{y}\left(t-t_{\tau}\right) \sin \alpha\right] \\
+\left(M_{34} \cos \alpha-M_{14} \sin \alpha\right)\left[W_{x}\left(t-t_{\tau}\right) \sin \alpha+W_{y}\left(t-t_{\tau}\right) \cos \alpha\right]
\end{array}\right\} \sum_{m} \delta\left(t-m t_{0}\right) .
\end{aligned}
$$

We should note that $D_{x}$ is not the $x$ component of the bunch dipole moment vector, rather it is the dipole moment projected along the rotated $x$ axis which would couple directly to the $x$ polarization of the dipole mode. The same applies to $D_{y}$.

From Eqs. (5), (6), (36), and (37) we get the BBU equations

$$
\begin{aligned}
& W_{x}(t)=\frac{N_{e} r_{0}}{\gamma}\left\{T_{11} \sum_{m} G_{x}\left(t-m t_{0}\right) W_{x}\left(m t_{0}-t_{\tau}\right)+T_{12} \sum_{m} G_{x}\left(t-m t_{0}\right) W_{y}\left(m t_{0}-t_{\tau}\right)\right\}, \\
& W_{y}(t)=\frac{N_{e} r_{0}}{\gamma}\left\{T_{21} \sum_{m} G_{y}\left(t-m t_{0}\right) W_{x}\left(m t_{0}-t_{\tau}\right)+T_{22} \sum_{m} G_{y}\left(t-m t_{0}\right) W_{y}\left(m t_{0}-t_{\tau}\right)\right\} .
\end{aligned}
$$


We have introduced the constants $T_{i j}$ to simplify the equations. They are defined as

$$
\begin{aligned}
& T_{11}=M_{12} \cos ^{2} \alpha+M_{34} \sin ^{2} \alpha+\frac{\left(M_{14}+M_{32}\right)}{2} \sin 2 \alpha, \\
& T_{12}=M_{14} \cos ^{2} \alpha-M_{32} \sin ^{2} \alpha+\frac{\left(M_{34}-M_{12}\right)}{2} \sin 2 \alpha,
\end{aligned}
$$

$$
\begin{aligned}
& T_{21}=M_{32} \cos ^{2} \alpha-M_{14} \sin ^{2} \alpha+\frac{\left(M_{34}-M_{12}\right)}{2} \sin 2 \alpha, \\
& T_{22}=M_{34} \cos ^{2} \alpha+M_{12} \sin ^{2} \alpha-\frac{\left(M_{14}+M_{32}\right)}{2} \sin 2 \alpha .
\end{aligned}
$$

For solutions of $W_{x}$ and $W_{y}$ with a harmonic time dependence with frequency $\Omega$ we obtain

$$
\left(\begin{array}{c}
W_{x}(t) \\
W_{y}(t)
\end{array}\right)=\frac{\pi e I}{\gamma m c \omega_{0}} \exp \left(-i \Omega t_{\tau}\right)\left[\begin{array}{cc}
T_{11}\left(Z^{\prime \prime} T^{2} / Q\right)_{x} H\left(\Omega, \omega_{x}\right) & T_{12}\left(Z^{\prime \prime} T^{2} / Q\right)_{x} H\left(\Omega, \omega_{x}\right) \\
T_{21}\left(Z^{\prime \prime} T^{2} / Q\right)_{y} H\left(\Omega, \omega_{y}\right) & T_{22}\left(Z^{\prime \prime} T^{2} / Q\right)_{y} H\left(\Omega, \omega_{y}\right)
\end{array}\right]\left(\begin{array}{c}
W_{x}(t) \\
W_{y}(t)
\end{array}\right)
$$

In order to have the problem manageable algebraically, we assume a single dipole mode with two polarizations of identical strength and frequency. Under these circumstances we find that BBU is completely independent of the rotation of the mode polarization as one might have guessed from the symmetry of the problem. Mathematically this follows from the fact that eigenvalues of the $2 \times 2$ matrix appearing in the BBU Eq. (48) are independent of $\alpha$. It is the result of the relation

$$
\begin{gathered}
T_{11}+T_{22}=M_{12}+M_{34} \\
\left(T_{11}-T_{22}\right)^{2}+4 T_{12} T_{21}=\left(M_{12}-M_{34}\right)^{2}+4 M_{14} M_{32} .
\end{gathered}
$$

Following the now familiar steps, we find the following expressions for the BBU threshold current.
First, we let

$$
M^{2} \equiv\left(M_{12}-M_{34}\right)^{2}+4 M_{14} M_{32}
$$

and consider three cases.

Case $M^{2}=0$ :

$$
I_{\mathrm{th}}=-\frac{4 E \omega}{e c\left(Z^{\prime \prime} T^{2} / Q\right) Q\left(M_{12}+M_{34}\right) e^{\omega t_{\tau} / 2 Q} \sin \omega t_{\tau}} .
$$

Case $M^{2}>0$ : the threshold current is the smaller of the two values

$$
I_{\mathrm{th}}=-\frac{4 E \omega}{e c\left(Z^{\prime \prime} T^{2} / Q\right) Q\left(M_{12}+M_{34} \pm M\right) e^{\omega t_{\tau} / 2 Q} \sin \omega t_{\tau}} .
$$

Case $M^{2}<0$ : the threshold current is the smaller of the two values

$$
I_{\mathrm{th}}=-\frac{4 E \omega}{e c\left(Z^{\prime \prime} T^{2} / Q\right) Q e^{\omega t_{\tau} / 2 Q\left\{\left(M_{12}+M_{34}\right) \sin \omega t_{\tau} \pm|M| \cos \omega t_{\tau}\right\}} .}
$$

It should also be obvious at this point that the BBU threshold expressions will involve $T_{i j}$ as defined above when the two polarizations are not completely degenerate, thereby exhibiting an explicit dependence on the rotation angle $\alpha$.

\section{THREE PASS MACHINE WITH BEAM OPTICS ROTATED BY 90}

We now consider a three pass machine with a single dipole mode with two polarizations of identical strength and also assume the following form of beam transfer matrices:

$$
M^{12}=\left(\begin{array}{cc}
0 & A \\
B & 0
\end{array}\right) \text { and } M^{23}=\left(\begin{array}{cc}
C & 0 \\
0 & D
\end{array}\right)
$$

For the BBU equations with the assumed harmonic dependency in time we get from Eqs. (11)-(13)

$$
\begin{aligned}
& W_{x}(t)=\frac{\pi e I}{\gamma m c \omega_{0}}\left(\frac{Z^{\prime \prime} T^{2}}{Q}\right)_{x} H\left(\Omega, \omega_{x}\right)\left\{\begin{array}{c}
M_{14}^{13} \exp \left[-i \Omega\left(t_{\tau 1}+t_{\tau 2}\right)\right] W_{y}(t) \\
+M_{12}^{23} \exp \left(-i \Omega t_{\tau 2}\right) W_{x}(t)+M_{14}^{12} \exp \left(-i \Omega t_{\tau 1}\right) W_{y}(t)
\end{array}\right\}, \\
& W_{y}(t)=\frac{\pi e I}{\gamma m c \omega_{0}}\left(\frac{Z^{\prime \prime} T^{2}}{Q}\right)_{y} H\left(\Omega, \omega_{y}\right)\left\{\begin{array}{c}
M_{32}^{13} \exp \left[-i \Omega\left(t_{\tau 1}+t_{\tau 2}\right)\right] W_{x}(t) \\
+M_{34}^{23} \exp \left(-i \Omega t_{\tau 2}\right) W_{y}(t)+M_{32}^{12} \exp \left(-i \Omega t_{\tau 1}\right) W_{x}(t)
\end{array}\right\} .
\end{aligned}
$$

For convenience we have introduced the definitions $t_{\tau 1}=t_{\tau}^{12}$ and $t_{\tau 2}=t_{\tau}^{23}$. This coupled equation can be diagonalized to find the eigenvalues. In doing so we find that we have to solve the equation 


$$
e^{t \Omega t_{\tau 2}}= \pm \frac{\pi e I}{\gamma m c \omega_{0}}\left(\frac{Z^{\prime \prime} T^{2}}{Q}\right) \lambda_{ \pm}(\Omega) H(\Omega, \omega)
$$

where

$$
\lambda_{ \pm}(\Omega)=\frac{M_{12}^{23}+M_{34}^{23}}{2} \pm\left[\begin{array}{c}
\frac{1}{4}\left(M_{12}^{23}-M_{34}^{23}\right)^{2} \\
+e^{-2 i \Omega t_{\tau 1}}\left\{M_{14}^{12} M_{32}^{12} e^{2 i \Omega t_{\tau 2}}+\left(M_{14}^{12} M_{32}^{13}+M_{32}^{12} M_{14}^{13}\right) e^{i \Omega t_{\tau 2}}+M_{14}^{23} M_{32}^{13}\right\}
\end{array}\right]^{1 / 2} .
$$

We then find the following expression,

$$
I_{\mathrm{th}}=-\frac{2 E \omega}{e c\left(Z^{\prime \prime} T^{2} / Q\right) Q e^{\omega t_{\tau} / 2 Q\left\{\operatorname{Re} M_{ \pm} \cdot \sin \omega t_{\tau}^{23}+\operatorname{Im} M_{ \pm} \cdot \cos \omega t_{\tau}^{23}\right\}},}
$$

where the threshold current is the smaller of the two values and where $\operatorname{Re} M_{ \pm}$and $\operatorname{Im} M_{ \pm}$are defined as

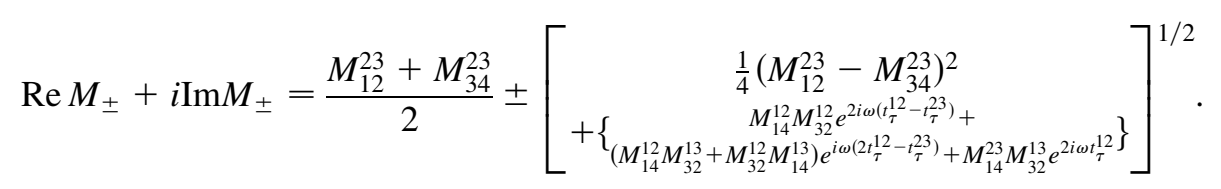

The dependency of the BBU threshold current on various beam transport parameters is already quite complex to analyze. Obviously, an analytic solution for even more general cases would be difficult to obtain.

\section{CONCLUSIONS}

From the BBU threshold formulas presented here one can see how and why a change in the BBU threshold current could occur in the case when the transverse beam optics are coupled. They also explain why the method devised by Rand and Smith [2] was effective in raising the BBU threshold limit in the JLAB IR FEL Upgrade. However, one has to keep in mind that the BBU threshold current can actually be decreased with this method depending on machine and beam parameters.

We note that the commonly used BBU threshold formula, Eq. (21), is not expected to be very accurate when $Q$ is not much greater than $\omega t_{\tau}$. Our second order expression, Eq. (23), gives a different expression for the threshold current in such a situation. Therefore, in a large machine with a circumference of several kilometers with heavily damped HOMs, one must be very careful in analytically estimating BBU thresholds. In addition, we have also derived analytical expressions for the threshold current in two special model cases. It is hoped that they should serve as useful models for studying the interdependence of multipass BBU instability on various beam and machine parameters.

We have also included a BBU threshold formula for a multipass machine, Eq. (27), from which one can derive a scaling behavior with the number of passes when the machine parameters are known. The formula can be applied to a microtron, for example.

\section{ACKNOWLEDGMENTS}

The author thanks K. Beard and C. Tennant for reading the manuscript. This work was supported by the
Department of Energy Contract No. DE-AC05-84ER40150.

\section{APPENDIX A: SPECIAL SOLVABLE CASES OF THE BBU EQUATION}

Let us rewrite Eq. (17) in Sec. II using Eqs. (14) and (15). We get

$$
\begin{aligned}
1+\xi^{2}-2 \xi \cos \omega_{x} t_{0}= & \frac{\pi e I}{\gamma m c \omega_{0}} \\
& \times\left(\frac{Z^{\prime \prime} T^{2}}{Q}\right)_{x} M_{12} \xi e^{-i \Omega t_{\tau}} \sin \omega_{x} t_{0} .
\end{aligned}
$$

Now let $t_{\tau}=m t_{0}$ where $m$ is the recirculation path length in time measured as multiples of the bunching period. In this paper we assume $m$ is an integer number. For multipass BBU instability with more general bunching schemes including the case of an energy recovery, see Ref. [9]. Noting that $e^{-i \Omega t_{\tau}}=e^{\left(\omega_{x} / 2 Q\right) t_{\tau}} \xi^{m}$ we obtain the following $(m+1)$ th order polynomial equation:

$$
\begin{aligned}
& \xi^{m+1}-2 \xi^{m} \cos \omega_{x} t_{0}+\xi^{m-1} \\
& =\frac{\pi e I}{\gamma m c \omega_{0}}\left(\frac{Z^{\prime \prime} T^{2}}{Q}\right)_{x} M_{12} e^{\left(\omega_{x} / 2 Q\right) t_{\tau}} \sin \omega_{x} t_{0} .
\end{aligned}
$$

We replaced $\xi$ by $1 / \xi$ for convenience and now note that $\xi=e^{\left(\omega_{x} / 2 Q\right) t_{0}} e^{i \Omega t_{0}}$.

For the case of either $m=1$ or 2 all roots of Eq. (A2) can be found analytically. Even though it may appear that these cases are mainly of a theoretical interest, it can actually be a realistic model for a small size recirculating accelerator operating with a sufficiently low bunch repetition rate. 
Case $m=1$ :

In this instance Eq. (A2) becomes

$$
\begin{aligned}
\xi^{2}-2 \xi \cos \omega_{x} t_{0}+1= & \frac{\pi e I}{\gamma m c \omega_{0}}\left(\frac{Z^{\prime \prime} T^{2}}{Q}\right)_{x} M_{12} e^{\left(\omega_{x} / 2 Q\right) t_{0}} \\
& \times \sin \omega_{x} t_{0} \equiv \varepsilon^{\prime} I .
\end{aligned}
$$

Solutions of (A3) are

$$
e^{i \Omega t_{0}}=e^{-\left(\omega_{x} / 2 Q\right) t_{0}}\left(\cos \omega_{x} t_{0} \pm i \sin \omega_{x} t_{0} \sqrt{1-\frac{\varepsilon^{\prime} I}{\sin ^{2} \omega_{x} t_{0}}}\right) .
$$

Consequently, we get an exact expression for the BBU threshold current

$$
I_{\mathrm{th}}=\left(1-e^{\left(\omega_{x} / Q\right) t_{0}}\right) \frac{E \omega_{0}}{\pi e c\left(\frac{Z^{\prime \prime} T^{2}}{Q}\right)_{x} M_{12} e^{\left(\omega_{x} / 2 Q\right) t_{0}} \sin \omega_{x} t_{0}} .
$$

For a high $Q$ mode where the approximation 1 $e^{\left(\omega_{x} / Q\right) t_{0}} \approx-\left(\omega_{x} / Q\right) t_{0}$ is valid (one should notice that this is the only condition required), we reproduce our first order threshold formula Eq. (21) presented in Sec. II.

Case $m=2$ :

In this case we have to solve a cubic equation in $\xi$ [10]. From Eq. (A2) we get

$$
\begin{aligned}
\xi^{3}-2 \xi^{2} \cos \omega_{x} t_{0}+\xi= & \frac{\pi e I}{\gamma m c \omega_{0}}\left(\frac{Z^{\prime \prime} T^{2}}{Q}\right)_{x} M_{12} e^{\left(\omega_{x} / 2 Q\right) t_{\tau}} \\
& \times \sin \omega_{x} t_{0} \equiv \varepsilon^{\prime} I
\end{aligned}
$$

First, we let

$$
\begin{aligned}
q & =\frac{1}{3}-\frac{4}{9} \cos ^{2} \omega_{x} t_{0} \quad \text { and } \\
r & =-\frac{1}{3} \cos \omega_{x} t_{0}-\frac{8}{27} \cos ^{3} \omega_{x} t_{0}+\frac{\varepsilon^{\prime}}{2} I .
\end{aligned}
$$

If $q^{2}+r^{2}>0$, Eq. (A6) has one real root and a pair of complex conjugate roots. If $q^{2}+r^{2} \leq 0$, all roots are real. Since $\xi=e^{\left(\omega_{x} / 2 Q\right) t_{0}} e^{i \Omega t_{0}}$ is complex, we require the condition $q^{2}+r^{2}>0$ for our solution.

If we define $s_{1}$ and $s_{2}$

$$
s_{1}=\left(r+\sqrt{q^{3}+r^{2}}\right)^{1 / 3}, \quad s_{2}=\left(r-\sqrt{q^{3}+r^{2}}\right)^{1 / 3}
$$

three roots of Eq. (A6) are given by

$$
\begin{gathered}
\xi_{1}=\left(s_{1}+s_{2}\right)+\frac{2}{3} \cos \omega_{x} t_{0}, \\
\xi_{2}=-\frac{1}{2}\left(s_{1}+s_{2}\right)+\frac{2}{3} \cos \omega_{x} t_{0}+i \frac{\sqrt{3}}{2}\left(s_{1}-s_{2}\right),
\end{gathered}
$$

$$
\xi_{3}=-\frac{1}{2}\left(s_{1}+s_{2}\right)+\frac{2}{3} \cos \omega_{x} t_{0}-i \frac{\sqrt{3}}{2}\left(s_{1}-s_{2}\right) .
$$

The root $\xi_{1}$ is real and the other pair of roots $\xi_{2}, \xi_{3}$ are complex conjugates.

Note that we have at the threshold current

$$
\left|\xi_{2}\right|=\left|\xi_{3}\right|=e^{\omega_{x} t_{0} / 2 Q} .
$$

Consequently,

$$
\begin{aligned}
e^{\omega_{x} t_{0} / Q}= & s_{1}^{2}+s_{2}^{2}-s_{1} s_{2}+\frac{4}{9} \cos ^{2} \omega_{x} t_{0} \\
& -\frac{2}{3}\left(s_{1}+s_{2}\right) \cos \omega_{x} t_{0},
\end{aligned}
$$

where $s_{1}$ and $s_{2}$ are defined at $I=I_{\text {th }}$. Therefore, Eq. (A13) can be solved to find an exact expression for the BBU threshold current. After some algebraic manipulations we obtain

$$
\begin{aligned}
I_{\mathrm{th}}= & \frac{E \omega_{0}}{\pi e c\left(Z^{\prime \prime} T^{2} / Q\right)_{x} M_{12} e^{\left(\omega_{x} / 2 Q\right) t_{\tau}} \sin \omega_{x} t_{0}} \\
& \times\left\{\begin{array}{l}
F_{ \pm}^{3}+\left(1-\frac{4}{3} \cos ^{2} \omega_{x} t_{0}\right) F_{ \pm} \\
+\frac{2}{3} \cos \omega_{x} t_{0}-\frac{16}{27} \cos ^{3} \omega_{x} t_{0}
\end{array}\right\},
\end{aligned}
$$

where

$$
F_{ \pm}=\frac{1}{3} \cos \omega_{x} t_{0} \pm \sqrt{e^{\left(\omega_{x} / Q\right) t_{0}}-\sin ^{2} \omega_{x} t_{0}} .
$$

Of course, the beam would be unstable if the average current exceeds the smaller of two (if both positive) currents given by Eq. (A14). We should also mention that one can show that Eq. (A14) reproduces our first order threshold formula Eq. (21) by carrying out an expansion in $\left(\omega_{x} / Q\right) t_{0}$ to first order under the assumption that $1-$ $e^{\left(\omega_{x} / Q\right) t_{0}} \approx-\left(\omega_{x} / Q\right) t_{0}$ and $\left(\omega_{x} / Q\right) t_{0}<4 \cos ^{2} \omega_{x} t_{0}$.

\section{APPENDIX B: ACCURACY OF THRESHOLD CURRENT PREDICTIONS}

In this appendix we compare our approximate formula Eq. (21) with the exact expression of the BBU threshold current Eq. (A14) in detail to assess the accuracy and the usefulness of approximate threshold current expressions presented in the paper.

First, comparing with Eq. (A5) the amount of error introduced in the estimation of $I_{\mathrm{th}}$ is the difference in value between $1-e^{\left(\omega_{x} / Q\right) t_{0}}$ and $-\left(\omega_{x} / Q\right) t_{0}$ [our first order formula Eq. (21) always underestimates the instability threshold current in this case). Note that all other dependence on various beam and machine parameters is completely identical.

For an illustration let us assume a dipole mode of frequency $1889 \mathrm{MHz}$ and $t_{0}=0.1 \mu$ s (i.e. $10 \mathrm{MHz}$ bunching frequency). The result of an error estimate which is a function of $Q$ is summarized in the Table II. 
TABLE II. An error estimate for threshold currents between Eqs. (A5) and (21) is summarized.

\begin{tabular}{lccc}
\hline \hline \multicolumn{1}{c}{$Q$} & {$\left[\exp \left(\omega_{x} / Q\right) t_{0}\right]-1$} & $\left(\omega_{x} / Q\right) t_{0}$ & $\%$ \\
\hline 1000 & 2.276886 & 1.18689 & 47.9 \\
10000 & 0.126020 & 0.118689 & 5.8 \\
100000 & 0.0119397 & 0.0118689 & 0.59 \\
$1 \times 10^{6}$ & $1.18760 \mathrm{E}-3$ & $1.18689 \mathrm{E}-3$ & 0.059 \\
$10 \times 10^{6}$ & $1.18696 \mathrm{E}-4$ & $1.18689 \mathrm{E}-4$ & $5.9 \mathrm{E}-3$ \\
$100 \times 10^{6}$ & $1.18690 \mathrm{E}-5$ & $1.18689 \mathrm{E}-5$ & $5.9 \mathrm{E}-4$ \\
\hline \hline
\end{tabular}

The percentage deviation of threshold current estimated with Eq. (21) from the true value provided by Eq. (A5) is listed in the fourth column.

Let us now turn to Eq. (A14). In this case an error occurs as a result of differences between two expressions

$$
\begin{aligned}
A_{ \pm} \equiv & F_{ \pm}^{3}+\left(1-\frac{4}{3} \cos ^{2} \omega_{x} t_{0}\right) F_{ \pm}+\frac{2}{3} \cos \omega_{x} t_{0} \\
& -\frac{16}{27} \cos ^{3} \omega_{x} t_{0}
\end{aligned}
$$

and

$$
B \equiv-\frac{\omega_{x} t_{0}}{2 Q \cos \omega_{x} t_{0}},
$$

where $F_{ \pm}$are given by Eq. (A15). Now the task of evaluating errors in estimation is more complicated as they depend on both parameters $Q$ and $\omega_{x}$. To start, Eq. (21) predicts that the beam is stable in a situation where a HOM frequency falls in frequency regions $2 n \pi<\omega t_{\tau}<(2 n+$ 1) $\pi$, where $n$ is an integer number for given $t_{\tau}$. In reality, the beam is not stable even for a dipole mode frequency in stop bands; we can see this from Eq. (A14). One can convince oneself that, generally, $I_{\mathrm{th}}$ is determined by an expression involving $F_{+}$for a frequency in stop bands, while it is determined by the threshold expression involving $F_{-}$in the complementary allowed frequency regions. Threshold current values for a frequency in stop bands are typically larger about by a factor $Q / \omega t_{0}$, which is a very large number for a typical dipole HOM in accelerating cavity. Therefore Eq. (21) provides still very useful information.

In Table III we show the $Q$ dependence of errors in threshold current estimates based on the first order formula for a typical HOM frequency of $1889 \mathrm{MHz}$ belonging to the allowed region. The bunching period, $t_{0}=0.1 \mu \mathrm{s}$ is same as in the case of $m=1$. A percentage deviation of threshold current estimated with Eq. (21) from the true
TABLE III. A typical error which one is expected to obtain by using Eq. (21) in estimating the BBU threshold current is computed from an exact expression (A14). The case of a $1889 \mathrm{MHz}$ dipole mode is shown.

\begin{tabular}{lccc}
\hline \hline \multicolumn{1}{c}{$Q$} & $A_{-}$ & $B$ & $\%$ \\
\hline 1000 & -2.95940 & -0.733540 & 75.2 \\
10000 & -0.0838415 & -0.0733540 & 12.5 \\
100000 & $-7.43347 \mathrm{E}-3$ & $-7.33540 \mathrm{E}-3$ & 1.3 \\
$1 \times 10^{6}$ & $-7.34515 \mathrm{E}-4$ & $-7.33540 \mathrm{E}-4$ & 0.13 \\
$10 \times 10^{6}$ & $-7.33638 \mathrm{E}-5$ & $-7.33540 \mathrm{E}-5$ & 0.013 \\
$100 \times 10^{6}$ & $-7.33550 \mathrm{E}-6$ & $-7.33540 \mathrm{E}-6$ & $1.3 \mathrm{E}-3$ \\
\hline \hline
\end{tabular}

value provided by Eq. (A5) is shown in the fourth column of Table III. We notice that an approximate threshold current obtained with Eq. (21) is in excellent agreement with the true threshold value when $Q \geq 10 \omega t_{\tau}$ or so. Even at very low $Q$ of about 1000 [we already know that Eq. (21) is not a good approximation for such a $Q$ which corresponds to $Q \approx \omega t_{\tau}$ ] the estimate is off only by a factor of 2 to 3 .

[1] C. Tennant et al., Phys. Rev. ST Accel. Beams 8, 074403 (2005).

[2] R.E. Rand and T. I. Smith, Part. Accel. 11, 1 (1980).

[3] B. C. Yunn, Jefferson Lab Report No. TN-05-032, 2005; Jefferson Lab Report No. TN-05-035, 2005.

[4] C. Tennant (private communication); E. Pozdeyev (private communication).

[5] R.E. Rand, Recirculating Electron Accelerators, Accelerators and Storage Rings Vol. 3 (Harwood Academic Publishers, New York, 1984); G. A. Krafft, J. J. Bisognano, and S. Laubach, Jefferson Lab Report No. TN-01-11, 1991.

[6] B. C. Yunn and L. Merminga, in Proceedings of the 1997 Particle Accelerator Conference, Vancouver, British Columbia, Canada, 1997 (IEEE, Piscataway, NJ, 1998), pp. 1694-1696.

[7] C. Tennant (private communication).

[8] E. Pozdeyev, Phys. Rev. ST Accel. Beams 8, 054401 (2005).

[9] B.C. Yunn, in Proceedings of the 1991 Particle Accelerator Conference, San Francisco, CA, 1991 (IEEE, Piscataway, NJ, 1991), pp. 1785-1787.

[10] Handbook of Mathematical Functions With Formulas, Graphs, and Mathematical Tables, edited by $\mathrm{M}$. Abramowitz and J.E. Stegun, National Bureau of Standards Applied Mathematics Series 55 (U.S. GPO, Washington, DC, 1972). 